\title{
Situation actuelle du français en Val d'Aoste
}

Enquête et résultats

\section{Tristan Hauff}

\section{(2) OpenEdition Journals}

Édition électronique

URL : http://journals.openedition.org/esp/1469

DOI : $10.4000 /$ esp. 1469

ISSN : 2532-0319

Éditeur

Centre d'Information sur l'Éducation Bilingue et Plurilingue

\section{Édition imprimée}

Date de publication : 1 décembre 2017

Pagination : 33-44

ISSN : 1127-266X

Référence électronique

Tristan Hauff, «Situation actuelle du français en Val d'Aoste », Éducation et sociétés plurilingues [En ligne], 43 | 2017, mis en ligne le 01 février 2019, consulté le 20 avril 2019. URL : http:// journals.openedition.org/esp/1469; DOI : 10.4000/esp.1469 


\section{SITUATION ACTUELLE DU FRANÇAIS EN VAL D'AOSTE. ENQUÊTE ET RÉSULTATS}

\section{Tristan HaUfF}

Basandosi sulla propria tesi di laurea, l'autore parla della situazione attuale in Valle d'Aosta, percepita soprattutto tramite interviste realizzate in loco. Le sue constatazioni sull'uso reale delle lingue si basano su statistiche recenti e sulle sue interviste. Viene esaminata la politica linguistica della regione per quanto riguarda l'istruzione al fine di conoscere le grandi linee dell'amministrazione regionale rispetto al francese e di rispondere alle seguenti domande: come avvenga concretamente la salvaguardia della lingua francese in una Valle d'Aosta italianizzata, quale sia le percezione delle proprie lingue da parte dei locutori e quali siano $i$ comportamenti linguistici adottati dai Valdostani nei confronti del francese.

Paole-chiave: Valle d'Aosta, inchiesta, politica linguistica, comportamenti, francese in VDA

Taking off from his Master's dissertation, the author discusses the linguistic situation in the Aosta Valley, as perceived in particular in the interviews he carried out there. He backs up his observations of the actual use of the languages by recent statistics and his interviewees' declarations. The Region's language policy both in education and administration is analyzed to get an idea of the general, official approach to French and to begin answering the following questions: in this Italianized Region, in what practical ways is the French language preserved; how speakers view their own languages; and what the attitudes towards French are, as expressed by the inhabitants themselves.

Keywords: par Keywords: Aosta Valley, survey, language policy, attitudes, preserving the French language.
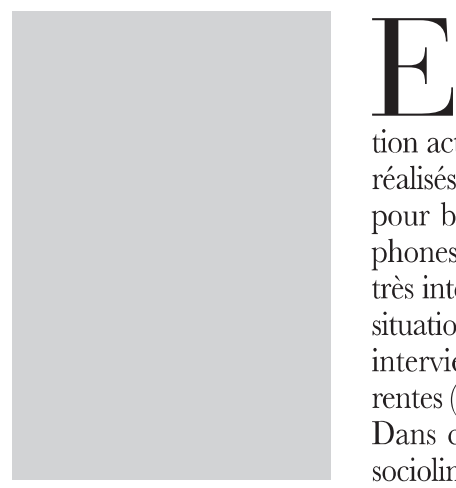

n marge de notre mémoire sur les aspects phonologiques et sociolinguistiques actuels du français en Vallée d'Aoste (VDA), nous allons présenter un chapitre traitant de la situation actuelle de la langue, perçue notamment au travers d'interviews réalisés sur place. Ces entretiens d'une trentaine de minutes, ayant pour but d'établir un profil sociolinguistique des locuteurs francophones, nous ont en outre permis de rassembler des informations très intéressantes sur les attitudes et visions des Valdôtains quant à la situation actuelle du français dans leur région. 24 personnes ont été interviewées, de sexes, âges, milieux et langues maternelles différentes (italien ou francoprovençal).

Dans cet article, nous allons dessiner les contours de la situation sociolinguistique actuelle en VDA, en mettant naturellement l'accent 
Situation actuelle du français en Val d'Aoste.

Enquête et résultats

T. HAUFF

USAGE RÉEL

ET STATISTIQUES:

L.ANGUE

VÉHICULAIRE OU

LANGUE DE

CONTAGT sur la langue française, en prenant en compte les points de vue des locuteurs francophones valdôtains interviewés. Nous ferons premièrement le constat de l'utilisation réelle des langues de la VDA en nous appuyant sur des statistiques récentes. Puis, nous parlerons de la politique linguistique de la région au niveau éducatif et de la vie publique pour connaitre les grandes lignes de l'administration régionale vis-à-vis du français et répondre à cette question : Dans une VDA italianisée, comment se déroule la sauvegarde de la langue française dans la pratique? Il convient donc aussi d'étudier le rapport direct qu'ont les locuteurs avec celle-ci: Sur quelles normes se base leur français en VDA, comment les locuteurs perçoivent-ils leur propre langue, et aussi quelles sont les attitudes linguistiques affichées par les Valdôtains quant au français.

Pour nous rendre compte de la présence réelle des langues majoritaires de la VDA (italien, francoprovençal et français), nous allons nous appuyer sur une enquête réalisée par la Fondation Émile Chanoux en 2003, se présentant comme «le premier recensement linguistique réalisé en VDA depuis 1921 (date du dernier recensement comportant une question sur la langue)». Cette enquête a pour but de «mettre en lumière la situation présente et la probable évolution future de la situation linguistique en VDA». En tout, 7250 questionnaires ont été renvoyés par des locuteurs de toutes tranches d'âge et de toutes communes de la Vallée. En premier lieu, nous pouvons remarquer que $87,79 \%$ des interrogés ont choisi de répondre à leur questionnaire en italien, contre $12,12 \%$ en français (pourcentages pondérés). Quant à la question de la langue maternelle, $71,58 \%$ des locuteurs déclarent avoir litalien comme langue première, $12,16 \%$ le francoprovençal et $0,99 \%$ le français $(1,09 \%$ des personnes interrogées sont nées en France). Ces premiers résultats sont assez catégoriques : Le français n'est ni la langue véhiculaire ni la langue maternelle des Valdôtains. On note tout de même la présence non-négligeable du francoprovençal: 12,16\% l'ont comme langue maternelle, et $67,35 \%$ affirment le connaitre. Cependant, il est important de s'intéresser à d'autres aspects linguistiques qui caractérisent le français en VDA outre l'usage courant. En effet, il est intéressant de noter la vision culturelle et patrimoniale qu'ont les Valdôtains du français: si on leur demande à quel groupe linguistique ils estiment appartenir, on s'aperçoit que la langue française apparait chez $8,4 \%$ des interrogés (en plus d'autres langues pour la quasi-totalité). Lorsqu'il s'agit de la question «En quelle langue lisez-vous livres, revues, journaux?», 23,94\% ont répondu «italien-français», ce qui confirme l'hypothèse que le français est bien présent sous d'autres formes que le véhiculai- 
Situation actuelle du français en Val d'Aoste.

Enquête et résultats

T. HAUFF

\section{POLITIQUE \\ LINGUISTIQUE/GLO TTOPOLITIQUE}

\section{ÉDUCATION}

re (31,80\% ont de plus déclaré regarder la télévision en «italien-français»). Au niveau de l'éducation, $54,01 \%$ choisiraient l'italien et le français comme langues d'enseignement, seuls $14,51 \%$ ne choisiraient que l'italien.

La question que l'on se pose est donc: La VDA est-elle bilingue? $78,36 \%$ disent «bien» ou «assez bien» comprendre le français, $58,06 \%$ affirment «bien» ou «assez bien» le parler, et les chiffres sont similaires quand il s'agit de le lire, et sont plus faibles lorsqu'il s'agit de l'écrire. Les conclusions que nous pouvons avancer à l'issue du sondage de la Fondation Chanoux sont les suivantes: le français n'est pas une langue véhiculaire, mais est connu par la majorité à des degrés différents. Il occupe donc une place spéciale dans l'espace linguistique et côtoie l'italien, qui est la langue dominante dans la grande majorité des domaines en VDA. Le francoprovençal, quant à lui, occupe une place importante dans les vallées reculées et est la langue d'usage d'une partie des Valdôtains. Dans beaucoup de communes de ces vallées, la majorité des locuteurs utilise l'italien et le francoprovençal comme langues de conversation. Exemple de la commune d'Introd (environ 600 habitants) à la question «Quelles langues et/ou quels dialectes utilisez-vous lorsque vous êtes avec vos amis?", $35,23 \%$ ont répondu italien - francoprovençal (Patois) et 26,94\% francoprovençal (Patois).

(http://www.fondchanoux.org/recherchelinguistique.aspx., 2013)

Après avoir pris connaissance du degré d'usage des trois principales langues de la VDA, nous allons nous pencher sur la gestion officielle du français. En effet, la Région administrative a un grand rôle à jouer dans la conservation des langues locales. L'italien ne semble pas avoir besoin de faire l'objet de politique particulière, étant la langue première de la majorité des Valdôtains, alors que le français, n'étant au contraire pas langue véhiculaire, ne s'acquiert généralement pas par le contact social spontané. Il est dit que «la fonction didactique de l'enseignement est de contribuer à organiser et développer ces acquisitions par des apprentissages systématisés» (Blanchet 2012: 164). Nous allons donc étudier le rôle de l'éducation dans le domaine de la glottopolitique (Blanchet 2012: 156), pour ensuite nous pencher sur le rôle de la vie publique, pour voir comment le français y est utilisé.

Si le français a un usage limité en VDA, la Région Autonome s'efforce de maintenir la langue française dans le paysage, notamment via l'éducation. Les recommandations données par le Conseil de L'Europe ayant pour but la généralisation de l'éducation bilingue et plurilingue, dans un document paru en 1998 (Les langues vivantes: 
Situation actuelle du français en Val d'Aoste.

Enquête et résultats

T. HAUFF apprendre, enseigner, évaluer), ont une grande influence. S’inspirant de ce texte, la stratégie éducative établie par la Région passe notamment par: «l'enseignement précoce des langues (italien et français en maternelle et l'anglais en primaire)», «l'usage véhiculaire des langues, particulièrement du français dans les disciplines» (Duc. Ed. 2001: 3). Selon l'article $39 \mathrm{du}$ Statut Spécial de la région, le français et l'italien sont enseignés à heures égales. En effet, l'école maternelle est régionalisée en 1972 avec pour ambition mixité et bilinguisme grâce à une méthode didactique spécifique. Des activités bilingues sont ainsi proposées (Cavalli 1998: 28). Le même article émet aussi une ligne directrice facultative:

«Certaines matières peuvent être enseignées en français». Cependant, il semble qu'il y ait un grand déséquilibre dans les écoles à ce sujet, car c'est à chaque établissement ou à chaque professeur de prendre l'initiative d'utiliser le français dans une autre matière, ce qui demande une bonne connaissance du français de la part du professeur (ce qui n'est pas systématique). Selon l'enquête sociolinguistique auprès des élèves de la dernière classe de l'enseignement obligatoire faite par Pascale Berticux pour l'Université de Liège en 1982, à l'école moyenne, $18,5 \%$ des élèves n'ont aucune matière enseignée en français, et $45,7 \%$ en ont $6 \mathrm{~h}$ par semaine. Ces statistiques sont en outre confirmées par une de nos témoins:

«À l'école primaire peut-être qu'ils apprennent plus l'anglais que le français» (JB, locutrice de langue maternelle franco-provençale (FRPR).

Puis concernant les efforts des professeurs pour inclure le français à l'école:

«il y a quelques-uns qui font plus, mais il y a beaucoup qui font rien» (JB, FRPR).

Le projet est là, les élèves sont compétents en français à des degrés différents, mais le constat de l'Institut Régional de Recherche Éducative (Costa, Sobrero 2006: 69) nuance la politique linguistique: «La langue reste valorisée linguistiquement mais n'est pas valorisée dans le domaine de culture générale, vie quotidienne, compréhensions de disciplines non-linguistiques». Ce constat nous montre bien que le français est solidement enraciné dans les écoles comme matière linguistique, mais que le manque d'utilisation dans d'autres domaines qu'à l'école est un problème majeur pour le bilinguisme de la Vallée. De ce fait, des attitudes négatives peuvent être une conséquence de cette situation comme le dit un témoin: «il y a pas mal de jeunes qui ont une attitude de refus du français. C'est peut-être parce que l'école n'arrive pas à le véhiculer, à le proposer d'une façon plus brillante, plus active peut-être» (IM, FRPR). 
Situation actuelle du français en $\mathrm{Val}$ d'Aoste.

Enquête et résultats

T. HAUFF

VIE PUBLIQUE

NORME, INSÉCURITÉ LINGUISTIQUE ET HYPERCORRECTION
Le bilinguisme est alors dans ce cas éducatif, ce qui veut dire «que l'école est le contexte de référence pour l'apprentissage et l'acquisition du français» (Decime \& Vernetto 2007: 46). Une vérité qui est appuyée par un locuteur: «le français reste désormais une langue pour les écoles» (GG, FRPR).

Au niveau de l'administration, la région doit recruter si possible des fonctionnaires connaissant le français. Ceux-ci doivent passer un examen de connaissances en français, qui d'ailleurs entraîne une prime de bilinguisme, ce qui suit un «principe généralisé de compétence bilingue» (Louvin 2014: 269). Cependant, il semble que l'utilisation du français soit assez restreinte dans l'administration (1), et il n'y a pas de loi empêchant un fonctionnaire de répondre en italien à une question posée en français. On parle alors d'un droit de compréhension et non d'un droit de réponse en langue choisie. (ibid.: 269). En politique, les lois concernant la Région sont publiées dans les deux langues, et celles votées par le Conseil de la Vallée peuvent quant à elles être directement traitées en français (ibid: :268). La parité est ainsi présente dans les textes fondamentaux de la Région, «tant pour la vie civile et les actes politiques et administratifs que pour la scolarisation» (Coste, Sobrero 2006: 69).

Tous ces choix de politique linguistique peuvent être considérés comme «in vitro», traduisant la gestion du plurilinguisme par les institutions. Louis-Jean Calvet (1993) développe cette idée: «Si les choix 'in vitro' prennent le contre-pied de la gestion in vivo (gestion par le peuple) ou des sentiments linguistiques du locuteur, il sera difficile d'imposer à un peuple une langue nationale dont il ne veut pas» (p. 112). Cette citation peut faire penser à la situation actuelle et future en VDA, car la gestion par le peuple n'y est pas effective. Cependant, les sentiments linguistiques des locuteurs sont plus complexes, et seront traités dans la section suivante.

La section précédente nous a permis d'avoir une idée plus claire des statuts des langues de la VDA et de la situation de déséquilibre actuelle. Le français, présent surtout comme langue écrite, est donc principalement visible dans l'administration et dans l'enseignement. Comme nous l'avons vu auparavant, le système éducatif est un des derniers vecteurs de la langue française, mais il est aussi l'artisan de la standardisation de cette dernière.

C'est une réalité qui n'est d'ailleurs pas spécifique à la VDA mais qui se retrouve fréquemment (Deckert \& Vickers 2011: 39). La standardisation est en d'autres termes une idéologie du standard valorisant l'uniformité de la langue, avec sa forme écrite comme forme aboutic 
Situation actuelle du français en Val d'Aoste.

Enquête et résultats

T. HAUFF
(Gadet 2007: 27). Les enseignants n'étant majoritairement pas de langue maternelle française, c'est donc souvent comme une langue étrangère et non comme une langue pré-acquise que le français est enseigné. Les élèves sont ainsi traités comme «linguistiquement vides », à «remplir» par le français standard de référence (celui de Paris et du nord de la France), et non par un quelconque «français régional». On peut mettre en parallèle la standardisation du français en VDA avec le cas français: «L'école française, par le biais des grammaires et des enseignants, tend à imposer à l'enfant une langue uniforme qu'il n'utilisera pas souvent ailleurs» (Boyer 1991: 22). Si cette citation est vraie surtout pour la forme écrite du français, elle s'applique aussi pour la forme orale en VDA. En effet, la standardisation touche aussi bien l'écrit que l'oral. Afin d'illustrer cette standardisation nous pouvons prendre pour exemple une citation survenue pendant une conversation avec un lycéen $(\mathrm{FC})$ :

«À l'école moyenne, je disais nonante et ma professoresse m'avait dit que c'était pas faux en Belgique et j'ai continué à dire nonante parce que ma professoresse nous disait pas que c'était faux alors quand je suis allé dans le supérieur, la professoresse m'a dit 'tu ne peux pas dire nonante' et moi j'avais passé trois ans à dire nonante» (FC, de langue maternelle italienne (IT)).

Cet exemple nous montre ainsi un défaut de connaissances du français régional en VDA et des variantes voisines de la part de deux enseignants ignorant que cette variante faisait partie du français régional pour beaucoup et qu'elle est encore utilisée dans le Valais suisse voisin. Dans ce cas-ci, l'élève se retrouve alors contraint par la suite d'utiliser la forme normalisée.

La normalisation du français en VDA entraine des répercussions négatives pour les locuteurs, dont l'hypercorrection et l'insécurité linguistique. Celles-ci sont liées par le fait que «l'hypercorrectionisme témoigne d'une insécurité linguistique» (Calvet 1993: 53). Lors des entretiens, nous pouvons en effet parfois observer une correction immédiate des chiffres «nonante, septante» par «quatre-vingt-dix, soixante-dix», qui sont des produits de la langue standard. Ces corrections sont majoritairement la raison non pas d'une recherche de forme prestigieuse mais plutôt de la forme «juste», et traduisent une crainte de «mal parler» (Py 2001: 47) ou d'être «condamnés à l'erreur» (Cavalli, Coletta 2003: 22). Pour reconnaître cette distance entre son propre idiolecte et le français «légitime», il faut donc que les locuteurs aient conscience de la différence, ce qui explique que la plupart de nos témoins ayant fait preuve d'insécurité linguistique soient ceux qui ont une bonne connaissance de la langue française, et ont tendance à sous-évaluer leur niveau face à une norme sociale 
Situation actuelle du français en Val d'Aoste.

Enquête et résultats

T. HAUFF

\section{ATTITUDES LINGUISTIQUES}

externe représentée comme exigeante. Nous pouvons donc dire qu’il existe deux facteurs majeurs de l'insécurité linguistique en VDA (Cavalli, Coletta 2003: 151):

I] Le sentiment de ne pas avoir accès à la norme

II] L'absence de variété vernaculaire

Ce contrôle assidu du français est une réalité en VDA, aussi bien au niveau du lexique, que de la qualité de l'expression, de la syntaxe, etc., et interroge l'avenir du français dans la région s'il n'existe pas de français non contrôlé vernaculaire présent dans l'espace linguistique. (Py 2001: 47).

Comme évoqué ci-dessus, le français fait l'objet d'une politique linguistique principalement «in vitro», administrée par les institutions, notamment éducatives. De ce fait, le français peut être perçu négativement par les locuteurs de la VDA, alors que d'autres le verraient comme une porte d'ouverture au monde, ou encore comme un symbole de leur identité. Nous allons à présent étudier ces différentes attitudes linguistiques que l'on trouve parmi les Valdôtains vis-à-vis du français. Nous allons nous appuyer sur les locuteurs interviewés sur le terrain ainsi que sur l'étude de l'Institut Régional de recherche éducative de la VDA en 2003 (Cavalli, Coletta et al. 2003).

Cette étude a synthétisé trois types d'attitudes principales face au français: Les attitudes esthétiques («le français est une belle langue»), les attitudes fonctionnelles (utilité sociale par exemple) et les attitudes didactiques («le français est plus ou moins difficile»), auxquelles nous ajouterons les attitudes historiques et identitaires («le français est une langue du patrimoine du VDA qui m'appartient», ou au contraire, «le français est une langue étrangère qui n'est pas la mienne»). Par exemple:

«Je n’ai pas une origine valdôtaine, donc non, je ne la vois pas (la langue française) comme une langue strictement liée à mes racines» (SM, IT)

«Ma langue c'est le francoprovençal. Le français c'est une langue, c'est comme l'italien pour moi. [...] pas ma langue. [...]» (EM, FRPR)

En ce qui concerne les jeunes écoliers, il semble que les attitudes positives face au français viennent surtout de la part des locuteurs patoisants qui ont moins de difficultés à apprendre le français que leurs camarades italophones, pour qui l'apprentissage est plus difficile. Là, on retrouve surtout une attitude didactique qui prédomine chez les plus jeunes qui sont en voie d'apprentissage linguistique:

«'ai beaucoup de copains de classe qui parlent le patois et qui n'ont pas beaucoup de problèmes avec le français parce que le patois c'est 
Situation actuelle du français en Val d'Aoste.

Enquête et résultats

T. HAUFF très semblable au français, mais j'ai aussi beaucoup de copains de classe qui sont, euh, comme moi, qui ne parlent pas le patois et ils ont des problèmes à l'écrire. Et nous, nous n'aimons pas la grammaire française parce que nous la trouvons très difficile, mais les personnes, qui, qui n'ont pas de problèmes avec ça aiment le français parce que c'est une langue qui est facile pour eux, et, que, c'est une langue en plus. [..] et toutes les personnes comme moi ne l'aiment pas vraiment» (FC, IT)

Un changement d'attitude survient souvent plus tard. Là, certains voient un certain côté pratique dans la connaissance du français du fait de la proximité de territoires francophones, donc comme une «langue d'échange», surtout utile pour le tourisme, ou pour la famille et amis francophones. Cette perception est vécue par une majorité de nos témoins qui la nomment comme une des raisons principales de l'importance de la présence du français en VDA.

«Je le vois comme une langue d'échange, ça veut dire, je l'utilise avec les étrangers, les touristes» (MC, IT).

«C'est très important encore parce que pour moi, c'est un atout parce qu'on est tout près de la France, tout près de la Suisse [...] C'est une langue d'échange, très vivante [...] ( HS, FRPR)

" C'est une langue que je parle avec des français, des francophones, ou sinon presque jamais» (MC, FRPR)

Les attitudes identitaires viennent quant à elles surtout de la part des locuteurs patoisants étant souvent plus ou moins engagés dans la conservation du patrimoine. Ceux-ci sont majoritairement des Valdôtains d'un certain niveau culturel qui se définissent pour certains comme des défenseurs du patrimoine, une minorité s'identifiant parfois à celle ayant protégé le français face au fascisme.

Nous pouvons montrer un exemple de cette attitude identitaire quand la question suivante a été posée à un locuteur:

«Comment se traduit votre attachement au Val d'Aoste, par quelles actions, quelles pensées?»

«Oui, déjà en défendant les langues, ces langues (le francoprovençal et français), euh, et parce que je suis persuadé que lorsqu'on perd une langue, on perd la civilisation dont la langue est l'expression. Quand tu parles italien, tu penses italien. [...] tu penses en tant qu'italien. Quand tu parles en français, tu penses en français, quand tu parles francoprovençal tu penses en valdôtain. C'est une représentation du monde qui est différente» (DF, FRPR)

D'autres témoins ont en outre exprimé une attitude similaire:

«Historiquement c'est notre langue» (CA, FRPR)

«Le français fait partie de tout ce qui appartient à la vallée d'Aoste» (JB, FRPR) 
Situation actuelle du français en Val d'Aoste.

Enquête et résultats

T. HAUFF
Une critique du système venant de ceux-ci qui a été relevée plusieurs fois est que le français est conservé pour sauvegarder le statut économique de la région, et non l'inverse. De plus, nous avons pu observer une critique même de la part de groupes engagés pour la sauvegarde du français:

«Même les politiciens de l'Union Valdôtaine [....] dans le discours officiel ils parlent français et entre eux ils parlent patois et italien» (HS, FRPR)

Les Valdôtains peuvent donc se diviser en quatre catégories suivant leurs attitudes linguistiques (catégories inspirées de l'étude de l'IRREVDA, 2003: 562):

Ceux qui se considèrent francophones en considérant le français et le patois comme des langues du Val d'Aoste

Ceux qui se considèrent patoisants, et voient le français et l'italien comme des langues imposées

Ceux qui se considèrent italiens, et voient le français comme une langue «utile» (enthousiastes à orientation internationale)

Ceux qui se considèrent italiens et voient le français comme une langue imposéc (sceptiques à orientation internationale)

Il existe donc plusieurs attitudes linguistiques (2), mais si nous nous appuyons sur le sondage de la Fondation Chanoux, il apparait que la majorité $(57,8 \%)$ affirment être fiers ou assez fiers de parler français, et environ 69\% affirment qu'il est important ou assez important de connaitre cette langue pour être valdôtain. D'autre part, on peut relever que plus de témoins interrogés ont répondu se sentir italien plutôt que de se sentir valdôtains et que seuls 2,01\% des interrogés ont répondu le français pour la langue à laquelle ils se sentent le plus attachés.

Donc, même si les attitudes divergent beaucoup, il semble que la majorité des Valdôtains voient dans le français un élément important de leur patrimoine, ou ayant du moins une certaine utilité. Si cette théorie s'applique surtout pour les Valdôtains ayant un sentiment identitaire prononcé, il semble que les immigrés étrangers ne voient pas le français comme une porte d'accès à l'intégration dans la société, et que de nombreux immigrés italiens voient quant à eux le français comme une langue qui leur est imposée (D'après nos expériences personnelles sur place relatées dans notre mémoire). Les attitudes négatives face au français semblent être courantes en VDA selon nos témoins qui déclarent:

«Il y a un refus du français assez net» (MCC, IT)

«Quand tu parles français on te regarde mal, quand tu parles patois encore pire» (MCF, FRPR)

«L'attitude 'à quoi ça sert'» (IM, FRPR) 
Situation actuelle du français en Val d'Aoste.

Enquête et résultats

T. HAUFF

CONCLUSION
Un témoin (JB, FRPR) nous a en outre fait part d'expériences personnelles où des moqueries subies à son encontre, voire des usignes de racisme» qui se sont fait entendre vis-à-vis du français. Notamment à l'Université d'Aoste où le personnel n'a pas été en mesure de répondre en français.

Malgré les $69 \%$ cités ci-dessus, il est donc important de contraster ce chiffre avec l'usage réel de la langue française aujourd'hui dans la région, c'est- à-dire très restreint, limité à des contextes bien précis, et qui observe un déclin constant depuis la période fasciste.

Dans cet article, nous avons donc mis la langue française de la VDA en contexte et en perspective. Nous pouvons tirer quelques conclusions clés: Le français n'est pas langue véhiculaire en VDA. Il est connu à des degrés différents par une grande partie des Valdôtains, mais seule une minorité, souvent patoisante, le maitrise vraiment. Cette maitrise est presque toujours accompagnée d'une «raison»: identitaire, professionnelle, familiale, etc. Néanmoins, le français reste une des deux langues officielles de la région et fait l'objet d'une politique linguistique presque obstinée, notamment dans l'enseignement afin de conserver la langue dans le paysage valdôtain. Cette politique entraine des attitudes négatives dans certains cas, où des Valdôtains perçoivent le français comme une imposition, au contraire d'autres résidents engagés pour la protection de celui-ci, perçu comme un élément clé de leur patrimoine.

Le français est à présent langue seconde pour une partie des Valdôtains seulement, et langue étrangère apprise par le biais scolaire pour beaucoup. Nous n'assistons plus à une coexistence des deux langues mais à une survie du français face à la suprématie linguistique de l'italien. Malgré cela, le français est toujours présent officiellement et reste visible sur la scène valdôtaine. Si sa forme écrite est la plus manifeste de nos jours, nous rappelons qu'environ $58 \%$ des Valdôtains ayant répondu au sondage de l'association Chanoux prétendent «bien» ou «assez bien» parler le français. Mais de quelle manière, et selon quelles normes? Si nous nous sommes efforcés de passer en revue ce sujet, il semble que certains Valdôtains ne savent pas où situer leur français. Un témoin s'interroge: «On a une connaissance du français mais je n'arrive pas à comprendre à quel niveau» (RM, FRPR).

Afin de contribuer à une meilleure prise de conscience et à la reconnaissance du français régional de la Vallée d'Aoste, nous avons entrepris, en plus du travail de sociolinguistique présenté en partie dans cet article, une étude phonologique visant à répertorier officiellement le parler oral des Valdôtains dans notre mémoire (Hauff 2016), qui 
Situation actuelle du français en Val d'Aoste.

Enquête et résultats

T. HAUFF

\section{BIBLIOGRAPHIE}

peut être consulté à l'adresse suivante:

https://www.duo.uio.no/handle/10852/51526

BERTIEAUX P. 1982. Description et analyse du Français en Vallée d'Aoste. Enquête sociolinguistique auprès des élèves de la dernière classe de l'enseignement obligatoire. Dissertation présentée pour l'obtention de la licence en philologie romane, Université de Liège.

BLANCHET P. 2012. La linguistique de terrain. Méthode et théorie. (Une approche ethnosociolinguistique de la complexité). $2^{\mathrm{e} m e}$ éd. Presses Universitaires de Rennes.

BOYER H. 1991. Éléments de sociolinguistique: Langue, communication et société, Paris, Dunod.

CALVET J.-L. 1993. La sociolinguistique, Que sais-je? 6ème éd. 2009. Paris, Presses Universitaires de France.

CAVALLI M. (éd.). 1998. Pensare e parlare in più lingue. Esperienze di insegnamento e di formazione in Valle d'Aosta. Aoste, IRRSAE Vallée d'Aoste. CAVALLI M., D. GOLETTA, L. GAJO, M. MATTHEY \& C. SERRA. 2003. Langues, bilinguisme et représentations sociales au Val d'Aoste. Aoste, IRRE de la Vallée d'Aoste.

COSTE D. 2001. Compétence bi/plurilingue et (in)sécurité linguistique. L'École Valdôtaine. Valle d'Aoste regione d'Europa: l'educazione bi/plurilingue, ponte verso la cittadinanza europea. (Cahier pédagogique supplément au nr 54). Aoste: 10-18.

COSTE D., A. SOBRERO, M. CAVALLI \& I. BOSONIN. 2006. Multilinguisme, plurilinguisme, éducation. Les politiques linguistiques éducatives. IRRE de la Vallée d'Aoste.

DECIME R. \& G. VERNETTO (éds.). 2007. Profil de la politique linguistique éducative. Vallée d'Aoste - Rapport Régional, Région Autonome du Val d'Aoste et Conseil de l'Europe.

DECKERT S.K. \& C.H. VICKERS. 2011. An Introduction to Sociolinguistics: Society and Identity. London, New York, Continuum Publishing Group.

FERGUSON G.A. 1959. Diglossia, Word (nr 15): 325-340.

Fondation Émile Chanoux. 2003. Une Vallée d'Aoste bilingue dans une Europe plurillingue. Aoste, Fondation Émile Chanoux.

GADET F. 2007. La variation sociale en français. Nouvelle édition revue et augmentée. Paris, Ophrys.

HAUFF T. 2016. Le français régional de la Vallée d'Aoste, Aspects sociolinguistiques et phonologiques, Mémoire de maitrise. Oslo, Universitetet i Oslo. LOUVIN R. 2014. Quelques notes sur le statut juridique du français et du francoprovençal en Vallée d'Aoste, Caleidoscopio. Cultura, politica, società. Scritti in memoria di Regine Laugier édité par BENVENUTO M.F. \& I.M. ROBINSON, Rubbettino Editore. 
Situation actuelle du français en Val d'Aoste.

Enquête et résultats

T. HAUFF

\section{SITE INTERNET}

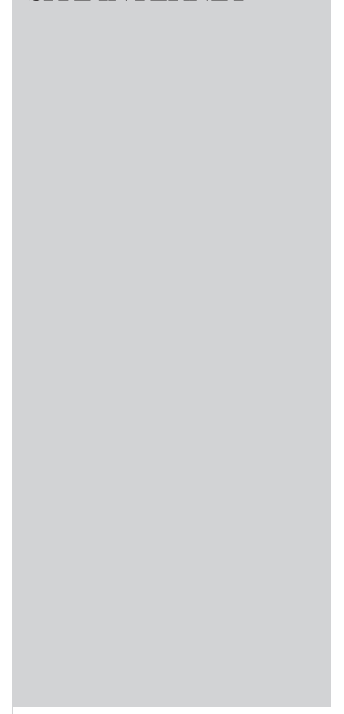

PY B., C. SERRA, L. GAJO \& D. COSTE. 2001. Regards extérieurs sur l'enseignement/apprentissage bilingue au Val d'Aoste. L'École Valdôtaine. Valle d'Aoste regione d'Europa: l'educazione bi/plurilingue, ponte verso la cittadinanza europea, Cahier pédagogique supplément au nr 54, pp. 44-58.

DUC V. (éd.). 2001. Premessa. L'École Valdôtaine. Valle d'Aoste regione d'Europa: l'educazione bi/plurilingue, ponte verso la cittadinanza europea, Cahier pédagogique supplément au nr 54, p. 3.

Fondation Chanoux. 2013. Sondage linguistique de la Fondation Émile Chanoux. Vu le 13 avril 2016. http://www.fondchanoux.org/sondagelinguistiqueq.aspx

\section{NOTES}

(1) En effet, le cas où un fonctionnaire de la Région n'a pas été en mesure ou a refusé de nous répondre en français durant le séjour sur le terrain ne fut pas inhabituel. Une réalité qui nous a été confirmée par une locutrice, qui nous a raconté plusieurs épisodes notamment à l'Université d'Aoste où le personnel n'a pas été en mesure de lui répondre en français, tout en subissant des moqueries pour ce choix de langue (épisode relaté plus bas dans l'article).

(2) Notons que les locuteurs interviewés dans le cadre de notre enquête sont tous francophones et ont pour la plupart une très bonne connaissance du français, ce qui implique souvent un engagement personnel dans l'apprentissage de la langue, traduit par des motivations identitaires, fonctionnelles ou encore familiales. 